\title{
Brain regions activated by endogenous preparatory set shifting as revealed by fMRI
}

\author{
H. A. SLAGTER \\ Duke University, Durham, North Carolina \\ and University of Amsterdam, Amsterdam, The Netherlands \\ D. H. WEISSMAN \\ University of Michigan, Ann Arbor, Michigan \\ B. GIESBRECHT \\ University of California, Santa Barbara, California \\ J. L. KENEMANS \\ Utrecht University, Utrecht, The Netherlands \\ G. R. MANGUN \\ University of California, Davis, California \\ A. KOK \\ University of Amsterdam, Amsterdam, The Netherlands \\ and \\ M. G. WOLDORFF \\ Duke University, Durham, North Carolina
}

\begin{abstract}
An ongoing controversy concerns whether executive control mechanisms can actively reconfigure the cognitive system in preparation for switching to a new task set. To address this question, we recorded brain activity from 14 healthy participants, using event-related functional magnetic resonance imaging, while they performed a cued attention task. Critically, in any particular trial, the cued task set was either the same as that in the previous trial or switched. As was hypothesized, cue-related, switchspecific preparatory activity was observed in a network of dorsal frontal and parietal brain areas that are typically associated with cognitive control processes. Moreover, the magnitude of switch-specific preparatory activity varied with the number of possible task sets that could be presented in a given trial block. These findings provide compelling support for the existence of top-down, preparatory control processes that enable set switching. Furthermore, they demonstrate that global task structure is a critical determinant of whether switch-specific preparatory activity is observed.
\end{abstract}

Models of cognitive control posit that the ability to flexibly adjust behavior in response to changing environmental conditions is fundamental to the achievement of behavioral goals (Baddeley, 1986; Norman \& Shallice, 1986). This requires changes in task set, the organization of mental resources necessary to accomplish a specific task (e.g., Monsell, 2003). A long-standing controversy

This research was supported by Dutch NWO Grant 42520206 to A.K. and J.L.K., a grant from the Psychology Research Institute of the University of Amsterdam to A.K., postdoctoral NSRA 1 F32 NS41867-01 to D.H.W., and by Research Grants R01-MH55714 and K05-MH02019 from the NIMH to G.R.M., PO1-NS41328 (Proj 2) from NINDS to M.G.W. and G.R.M, and RO1-MH60415 from the NIMH to M.G.W. We thank Ben Cheng and Allen Song for their assistance in data collection. Correspondence concerning this article should be addressed to M. G. Woldorff, Center for Cognitive Neuroscience, Duke University, Box 90999, LSRC Bldg., Room B203, Durham, NC 27708-0999 (e-mail: woldorff@duke.edu). involves the question of whether the cognitive system can be actively reconfigured in preparation for performing a new task (Altmann, 2003; Monsell, 2003). In line with this view, several behavioral studies have demonstrated that increasing the time between an instructional task cue and a subsequent target stimulus minimizes performance decrements (i.e., switch costs) for trials requiring a switch in task set (switch trials), in comparison with trials in which no switch in task set is required (repeat trials; Meiran, 1996; Rogers \& Monsell, 1995). However, recent behavioral studies have suggested that the effects of preparation on switch costs are due largely to the additional time needed to encode a new instructional cue, regardless of whether a switch in task set occurs (Altmann, 2004; Logan \& Bundesen, 2003). Thus, it is still unclear whether control processes can actively prepare the system in advance to switch to performing a new task. 
To resolve this issue, we used event-related functional magnetic resonance imaging (fMRI) to directly measure cue-triggered, switch-specific preparatory processes whose existence in behavioral paradigms is typically inferred only by the indirect measure of performance on target stimuli. Although set switching has been associated with greater activity in a network of frontal and parietal regions thought to implement cognitive control processes, prior fMRI studies have not resolved the issue of whether it is possible to prepare in advance for a set switch (Braver, Reynolds, \& Donaldson, 2003; Dreher, Koechlin, Ali, \& Grafman, 2002; Forstmann, Brass, Koch, \& von Cramon, 2005; Kimberg, Aguirre, \& D’Esposito, 2000; Rushworth, Paus, \& Sipila, 2001; Shulman, d'Avossa, Tansy, \& Corbetta, 2002; Smith, Taylor, Brammer, \& Rubia, 2004; Sohn, Ursu, Anderson, Stenger, \& Carter, 2000; Yantis et al., 2002). For example, in several studies, switch and repeat trials were presented in separate blocks, thereby confounding any possible preparatory switch-related activity with nonspecific effects, such as task difficulty, arousal, or strategy (e.g., Shulman et al., 2002; Sohn et al., 2000). In other studies, in which switch and repeat trials occurred randomly within the same blocks, the designs did not allow for the isolation of cue-related, switchspecific activity (e.g., Brass \& von Cramon, 2004; Forstmann et al., 2005; Rushworth et al., 2001; Smith et al., 2004; Yantis et al., 2002). For example, in the study by Brass and von Cramon (2004), a double-cue design was used in which the second cue could indicate a task that was the same as or different from that indicated by the first cue, and two cues were assigned per task. The goal was to separate activity related to cue switches from that related to set switches. However, because a target stimulus always followed very quickly after the second cue and because no cue-only trials were included, it was not possible to separate cue-related from target-related switch-specific activity in the hemodynamic response. In order to demonstrate that executive processes actively reconfigure the cognitive system in advance of a set switch, it is critical to demonstrate switch-related activity that is specifically triggered by the cue, as opposed to the target.

In several recent fMRI studies investigating set switching, cue-related preparatory activity in frontal and parietal areas has been isolated using event-related designs in which switch and repeat trials were presented within the same trial block (Barber \& Carter, 2005; Brass \& von Cramon, 2002; Luks, Simpson, Feiwell, \& Miller, 2002; Ruge et al., 2005; Shulman et al., 2002). In all but one of these studies (Barber \& Carter, 2005), no set-switch-related activity was observed during the cue period. This general pattern of results suggests that no additional executive control is recruited on switch trials during a preparation interval, even though the results from behavioral studies suggest that during the preparation interval, a new task set may need to be activated and an old task set may need to be inhibited (Allport, Styles, \& Hsieh, 1994; Meiran, 1996; Rogers \& Monsell, 1995). The one exception to these findings is the recent study by Barber and Carter that reported an effect of set switching on preparatory activity, which was observed only in the precuneus. This result would be consistent with the hypothesis that it is possible to prepare in advance for an upcoming set switch. However, as will be discussed below, we hypothesize that other regions should also be involved in preparatory aspects of set switching. We further hypothesize that these additional regions may not have been activated in the study of Barber and Carter or other previous studies, due to the global task structures of the experimental designs.

A possible explanation for the lack of switch-specific preparatory activity in prior fMRI studies may stem from the global task structure that was used. That is, aspects of the task designs may have induced strategies that minimized the utility of preparatory task set switching. Consistent with this view, recent behavioral findings indicate that global task structure may modulate the recruitment of switchspecific preparatory processes in set-switching paradigms (Mayr \& Kiegl, 2003). First, including a large number of randomly intermixed task sets within a trial block necessarily reduces the probability of set repetition, which could influence the strategy that is used to perform the task. For example, when numerous task sets are included in a block, subjects might strategically abandon the task set used in the previous trial, since it is unlikely to be used in the current trial. If such a strategy was being employed, both repeat and switch trials would then require the subjects to direct more extended processing toward encoding the task cue and retrieving the relevant stimulus-response mappings from memory, thereby making it more difficult to observe differences in cue activity between switch and repeat trials (Altmann, 2003; Logan \& Bundensen, 2003; Mayr \& Kiegl, 2003). Second, the preparation times (i.e., cue-target intervals) and intertrial intervals in previous event-related fMRI studies were typically several seconds or more in duration, considerably longer than those used in the behavioral literature. Such parameters may have reduced demands on set-switching processes and, hence, the probability of observing switch-related activity, because (1) long cue-target intervals allow greater time to prepare for a set switch (e.g., Meiran, 1996) and (2) greater intertrial intervals might allow inhibition directed toward task sets used in previous trials to dissipate (Mayr \& Keele, 2000). Therefore, evidence for switch-specific cue activity might be stronger in a faster paced task that more closely matched those used in previous behavioral studies.

In the present study, we used a fast-paced set-switching paradigm in combination with event-related fMRI to investigate (1) whether brain areas involved in set switching are activated in preparation for a set switch and (2) whether varying the number of task sets in a trial block modulates brain activity associated with switch-specific preparation. In each trial of our task, the participants were cued to prepare to discriminate the orientation of an upcoming rectangular-shaped target on the basis of either its color (yellow or blue) or its location (left or right; see Figure 1). In many trials, a target display followed cue presentation, and the subject's task was to indicate the orientation of the 


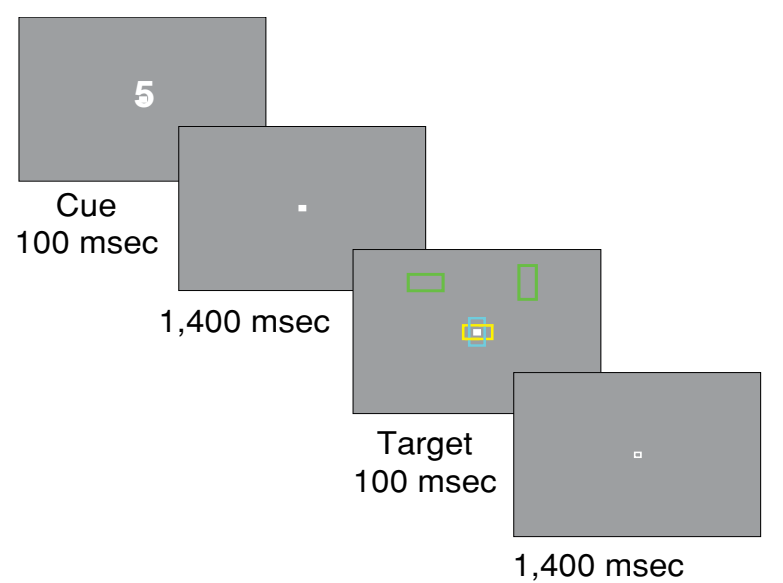

Figure 1. Example of a cue-plus-target trial. The cue instructed the subject to indicate the orientation (horizontal or vertical) of the rectangle with the cued feature (either blue, yellow, or left or right) in the upcoming target stimulus display.

cued rectangle (vertical or horizontal) within that display. In some trials, however, no target display followed cue presentation, which allowed us to isolate cue-related preparatory activity. In different trial blocks, the attentional cue was the same for each trial (repeated task block), switched randomly between two predefined features (e.g., left and right) of the same dimension (single-dimension task block), or switched randomly between all four features across the two dimensions of color and location (mixed-dimension task block).

This design afforded the investigation of two key questions about the neural mechanisms that enable us to adopt a new task set. First, within a block of trials, comparing the brain responses to cues instructing the subjects to switch task sets with those to cues instructing them to repeat a task set allowed us to investigate the neural mechanisms that help reconfigure the cognitive system to use a new task set during the cue-target interval. Second, comparing cuerelated brain responses on switch and repeat trials across the different types of trial blocks (repeated, single dimension, and mixed dimension) permitted us to investigate whether increasing the number of possibly relevant task set features and dimensions in a block reduces the magnitude of switch-specific preparatory activity, consistent with prior suggestions in the behavioral literature that global task context modulates the recruitment of preparation-related set-switching processes (Mayr \& Kiegl, 2003).

\section{METHOD}

\section{Subjects}

Fourteen healthy subjects (mean age, 23.4 years; 7 of them male) were recruited from the Duke University community and gave informed consent in accordance with the guidelines set by the Duke University Medical Center Institutional Review Board. All the subjects were right-handed, had normal or corrected-to-normal vision and had no history of neurological trauma or disorders.

\begin{abstract}
Apparatus
A PC computer was used for stimulus presentation and for the recording of response data. The stimuli were viewed through an MRcompatible, fiber-optic goggle system. Responses were recorded with an MR-compatible response box. The timing of the stimuli and the recording of the responses were controlled by commercially available software (Presentation, Neurobehavioral Systems).
\end{abstract}

\section{Event-Related fMRI Paradigm}

We used a cued attention paradigm in combination with a recently developed fast-rate event-related fMRI approach (Shulman et al., 1999; Weissman, Mangun, \& Woldorff, 2002; Woldorff et al., 2004). At the beginning of each 3 -sec trial, the subjects viewed a centrally presented cue (i.e., a single digit: $3,4,5$, or 6; visual angle, $0.28^{\circ} \times 0.38^{\circ}$; duration, $100 \mathrm{msec}$ ) that instructed them to attend to a rectangle at a particular spatial location (i.e., left or right) or to a centrally presented rectangle of a particular color (i.e., yellow or blue) within an upcoming target display (Figure 1). To prevent physical differences among the cue stimuli from confounding the results, we counterbalanced (across subjects) which target feature the subjects were instructed to attend to when a given cue stimulus was presented (i.e., for some subjects, a " 3 " cue meant attend left, whereas for others, it meant attend right, and so on). In cue-plustarget trials (33\%), the target display appeared 1,500 msec after cue onset and consisted of four rectangles: two green peripheral rectangles presented $4^{\circ}$ horizontally and $3.5^{\circ}$ vertically from fixation in the upper left and upper right visual fields and two overlapping, centrally presented rectangles of which one was yellow and the other blue (target duration $=100 \mathrm{msec}$ ). The subjects' task was to indicate the orientation (i.e., horizontal or vertical) of the rectangle with the cued feature by pressing one of two buttons with their right index or middle finger. To equate the difficulty of the color and location tasks, the aspect ratio of the vertical and horizontal axes of the rectangles was adjusted for each run on the basis of the performance in the previous run, separately for the peripheral (location task) and central (color task) rectangles. To compensate for the lower visual acuity in the periphery, the size range of the peripheral rectangles where the location task was performed $\left(1.75^{\circ} \times 1.25^{\circ}\right.$ to $3.13^{\circ} \times$ $1.25^{\circ}$ ) was greater than the size range of the central rectangles where the color task was performed $\left(0.88^{\circ} \times 0.63^{\circ}\right.$ to $\left.1.56^{\circ} \times 0.63^{\circ}\right)$. All the stimuli were presented on a dark gray background.

In cue-only trials $(33 \%)$, the cues were not followed by a target. We also included catch-cue-only trials $(8.3 \%)$, in which a fifth type of digit cue ("0") was presented for $100 \mathrm{msec}$. The subjects were instructed to press a (third) button with their right ring finger as quickly as possible upon presentation of this catch cue. Catch-cueonly trials were randomized into the trial sequence to ensure that the subjects kept identifying the cues in the repeated task blocks (see below). Finally, so-called no-stim trials containing only a fixation point $(25 \%)$ were included in order to allow subtraction of the average hemodynamic response to each of the other trial types in our fast-rate design (Burock, Buckner, Woldorff, Rosen, \& Dale, 1998; Woldorff et al., 2004). Within each 72-trial run, cue-plustarget, cue-only, catch-cue-only, and no-stim trials were presented in a pseudorandom order, so that, on average, each trial type was preceded by the same event distribution (Buckner et al., 1998; Burock et al., 1998; Woldorff, 1993; Woldorff et al., 2004).

In all the trials, the fixation point (a hollow, white square: $0.09^{\circ} \times$ $0.09^{\circ}$ of visual angle) was filled in as soon as the cue stimulus was presented. The fixation point remained filled during cue-plus-target trials until the offset of the target display (duration of fill $=1,600 \mathrm{msec}$ ) or until the equivalent time in cue-only trials. The reversion to a hollow fixation point at the same time in cue-plus-target and cue-only trials served to signal the end of the trial and was performed to equate the duration of cue-triggered attentional-orienting processes in cueplus-target and cue-only trials (e.g., Corbetta, Kincade, Ollinger, Mc- 
Avoy, \& Shulman, 2000). In no-stim trials, the hollow fixation point remained at fixation for the entire trial. The subjects were not aware of the no-stim trials since, to the subjects, these were only periods of slightly longer delay between subsequent cued trials, yielding the impression that the intertrial interval was variable.

Three types of trial blocks were used to investigate the neural mechanisms underlying set switching: repeated, single dimension, and mixed dimension. In repeated task blocks, the attention-directing cue instructed the subjects to attend to exactly the same stimulus feature in every trial (e.g., attend blue), and thus, no switching was required. In single-dimension task blocks, the cue randomly (i.e., from trial to trial) instructed the subjects to attend to the rectangle with either of two predefined features within the same stimulus dimension (i.e., blue or yellow in some blocks, left or right in other blocks). Since the cue types occurred in random order, in approximately $50 \%$ of the trials, the task set repeated (e.g., attend blue trial followed by attend blue trial), whereas in the other $50 \%$ of the trials, the subjects needed to switch between two features of the same dimension (e.g., attend blue trial followed by attend yellow trial). In mixed-dimension task blocks, the cue randomly instructed the subjects to attend to a rectangle with any one of the four possible stimulus features across both stimulus dimensions (i.e., blue, yellow, left, or right). Accordingly, in $25 \%$ of the trials, the task set repeated (e.g., attend blue trial followed by attend blue trial), in $25 \%$ of the trials, the subjects needed to switch between two features of the same dimension (e.g., attend blue trial followed by attend yellow trial), and in $50 \%$ of the trials the subjects needed to switch between two features belonging to different dimensions (e.g., attend blue trial followed by attend left trial). Thus, the global task context differed in all three types of task blocks, because these blocks varied in (1) the number of possibly relevant features and dimensions in the cued set and (2) the probability of a set switch. Note, however, that although the criteria used for rectangle selection varied across (and sometimes within) blocks, the rules for responding never changed.

\section{Procedure}

The subjects participated in four sessions: a training session, two fMRI sessions, and an ERP session. During the training session, the subjects performed an example of each type of task block four times in order to become familiar with the specific task requirements and to ensure that they were able to maintain fixation. During training, horizontal and vertical eye movements were monitored with two bipolar electrodes placed, respectively, on the left and right of the outer canthi and above and below the left eye. Electrode impedances were maintained below $10 \mathrm{k} \Omega$. The two electrooculogram (EOG) channels were continuously recorded with a band-pass filter of $0.01-100 \mathrm{~Hz}$, a gain of 1,000 (SynAmps I amplifiers from Neuroscan, Inc.), and digitized with a sampling rate of $250 \mathrm{~Hz}$. Recordings took place in an electrically shielded, sound-attenuated, dimly lit room.

After training, the subjects took part in two separate fMRI sessions and an ERP session that were all conducted on separate days. The interval between the two fMRI sessions was generally 2-4 weeks. During one of the fMRI sessions, the subjects performed the repeated and single-dimension task blocks. There were eight repeated task block runs, two for each of the four stimulus features (i.e., blue, yellow, left, and right). There were also eight single-dimension task block runs, four in which the subjects were cued randomly to attend to a single feature of the color dimension (i.e., attend blue or attend yellow) and four in which they were cued randomly to attend to a single feature of the location dimension (i.e., attend left or attend right). The order of the repeated and single-dimension task block runs was counterbalanced across subjects, with the restriction that the color and location task blocks were always grouped together; that is, half of the subjects got the color task blocks first, whereas the other half of the subjects got the location task blocks first. During the other fMRI session, the subjects performed eight runs of the mixed-dimension task block. The order of the two fMRI sessions was counterbalanced across subjects. During the ERP session, the subjects performed only the repeated and single-dimension task blocks. The order of the ERP session and its corresponding fMRI session was also counterbalanced across subjects. The present report is focused on the results from the fMRI studies. Results from the ERP session will be reported elsewhere.

\section{fMRI Data Acquisition}

Functional images were acquired on a General Electric 4-T scanner using an inverse spiral imaging sequence $(\mathrm{TR}=1.5 \mathrm{sec}, \mathrm{TE}=$ $31 \mathrm{msec}$, flip angle $=60^{\circ}$ ). During every task block, 164 brain volumes were collected, each of which contained 32 contiguous, 3.75mm-thick slices (in-plane resolution: $3.75 \times 3.75 \mathrm{~mm}$ ). Structural images were collected using a T1-weighted spin echo sequence $(\mathrm{TR}=12.2 \mathrm{msec}, \mathrm{TE}=5.3 \mathrm{msec}$, inversion time $=300 \mathrm{msec}$, flip angle $=20^{\circ}$ ). The first six functional images of each run contained no trials and were discarded prior to analysis of the functional data.

\section{Behavioral Data Analysis}

Each cue-plus-target trial was categorized as instructing the subjects to attend to the rectangle with (1) the same feature that was cued in the previous trial, (2) a different feature in the same dimension, or (3) a different feature in a different dimension. Each cue-plus-target trial was also classified according to whether it was presented in a repeated, single-dimension, or mixed-dimension task block. The effects of set switching on behavioral performance were measured using reaction times, error rates, and omitted response rates from the mixed-dimension and single-dimension task blocks. For the mixed-dimension task block, reaction times, error rates, and omitted response rates were analyzed separately with a repeated measures ANOVA. Each ANOVA contained the within-subjects factor of switch (repeat, switch within dimension, or switch across dimensions). Prior to these analyses, error rates and omitted response rates were arc-sin transformed to adjust the data to a normal distribution as assumed by an ANOVA (Snedecor \& Cochran, 1989). The behavioral data from the single-dimension task block were also entered into similar repeated measure ANOVAs, but the switch factor had only two levels: repeat and switch within.

Effects of the number of task set features and/or dimensions in a block were evaluated by determining whether performance on repeat and switch-within trials varied as a function of task block (repeated, single dimension, or mixed dimension). Two separate repeated measures ANOVAs were performed, one for response times on repeat trials and one for response times on switch-within trials, each with the within-subjects factor of task block. For repeat trials, task block had three levels (i.e., repeated, single dimension, or mixed dimension), whereas for switch-within trials, this factor had two levels (i.e., single dimension or mixed dimension).

To confirm that the subjects were identifying the cue stimuli in the repeated task blocks, as well as in the other task blocks, the response times and the omitted response rates for catch cues were entered into separate repeated measure ANOVAs with the within-subjects factor of task block (repeated, single dimension, or mixed dimension). In all of the above analyses, the Greenhouse-Geisser correction was applied where appropriate, and only corrected $F$ values will be reported.

\section{fMRI Data Analysis}

\section{Image Processing}

The software analysis package SPM'99 (Friston et al., 1995) was used to correct functional images for asynchronous slice acquisition and head motion, to normalize functional images to the Montreal Neurological Institute standard space, and to spatially smooth the functional data with a Gaussian filter (full-width half maximum $=$ $8 \mathrm{~mm}$ in the $x, y$, and $z$ dimensions). 


\section{Event-Related Analyses}

Selective averaging. Selective averaging was used to estimate the average hemodynamic response produced by each trial type (see Buckner et al., 1998). Each cue-only trial was categorized as instructing the subjects to attend to the rectangle with (1) the same feature as that cued in the previous trial, (2) a different feature in the same dimension, or (3) a different feature in a different dimension. Each cue-only trial was also classified according to whether it was presented in the (1) repeated task block, (2) singledimension task block, or (3) mixed-dimension task block. Each no-stim trial was categorized in exactly the same manner. Subsequently, the average event-related time course (starting $1.5 \mathrm{sec}$ before and ending $13.5 \mathrm{sec}$ after trial onset) was calculated for each trial type, separately at every voxel and for each subject. These average responses were converted to units of percent change from baseline, which we defined as the average of the signals present at trial onset and the immediately preceding time point. To remove overlap in the hemodynamic responses from adjacent trials caused by the fast-rate presentation of the trials, we subtracted from each cue-only response the average response for the no-stim trial type that was preceded by the same event distribution (Buckner et al., 1998; Burock et al., 1998; Woldorff et al., 2004).

Voxelwise analyses. To investigate the brain regions activated in each condition, each of the six cue-only time courses above was entered into a voxel-level, one-way repeated measures ANOVA. Activated regions were determined by a main effect of MR frame [eight

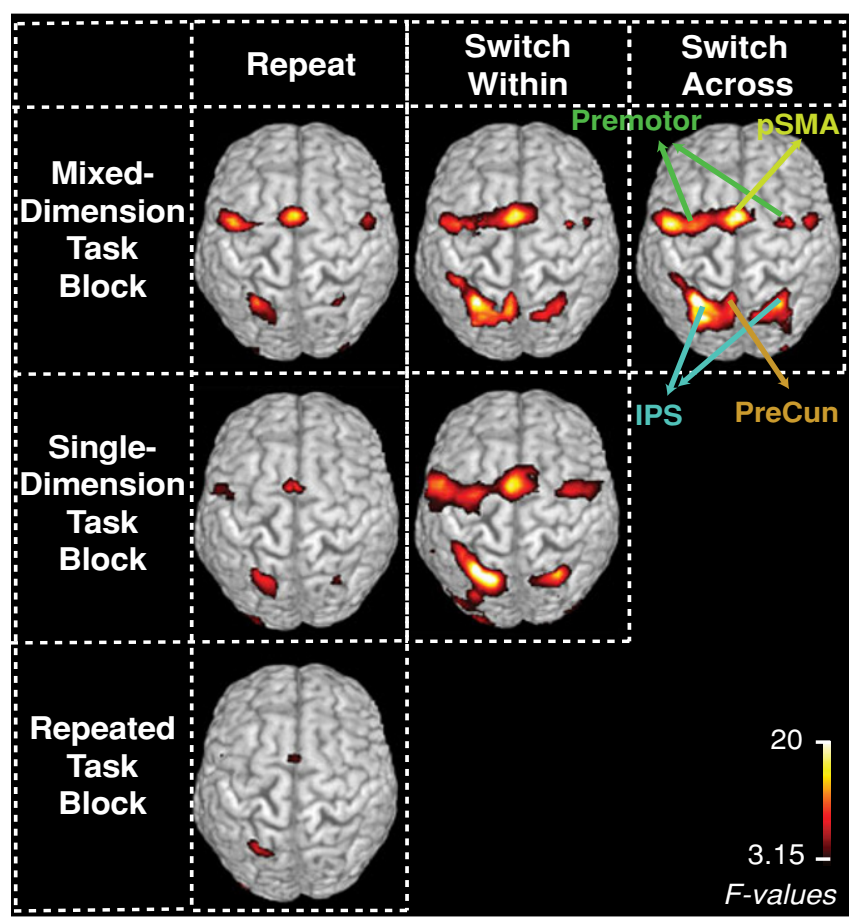

Figure 2. Brain areas showing cue-related activity. Cuerelated activity for repeat, switch-within, and switch-across trials is shown in the repeated, single-dimension, and mixeddimension task blocks. Note the increase in activation of the frontoparietal network (1) as a function of set-switching demands within the single-dimension and mixed-dimension task blocks and (2) as demands on control processes increased across the repeated, single-dimension, and mixed-dimension task blocks. pSMA, presupplementary motor area; IPS, intraparietal sulcus; PreCun, precuneus. frames $(1.5-13.5 \mathrm{sec}) ; F(7,91)=3.15, p<.005$; extent threshold of eight voxels]. All types of cues, regardless of condition, activated a highly overlapping network of brain areas (see Figure 2). Therefore, we averaged the overlap-corrected responses to cue-only trials across all conditions. In this way, one average hemodynamic response, reflecting the overall cue-related response collapsed across all cue types (except for the catch cues), was derived at every voxel and for each subject. To examine which areas were activated by cues in general, the average responses from the different subjects were entered into a voxel-level, one-way repeated measures ANOVA. As in our initial analysis, activated regions were determined by a main effect of MR frame [eight frames $(1.5-13.5 \mathrm{sec}) ; F(7,91)=3.15$, $p<.005$; extent threshold of eight voxels].

ROI analyses. The activations corresponding to the average cueonly trial response above were used to functionally define regions of interest (ROIs), in which we more directly assessed whether switchspecific preparatory activity was present. Each ROI was centered on a local maximum in the activation map for the average cue-related response and consisted of a $3 \times 3 \times 3$ cube of voxels. In every ROI, the overlap-corrected response for each of the six cue-related responses (i.e., the response after subtracting the no-stim; see above) was derived and averaged across all voxels within the ROI for each subject separately. Each subject's responses served as dependent measures in subsequent analyses that identified (1) the regions involved in switch-specific preparation and (2) the effects of global task context on switch-specific preparatory activity.

Switch-specific preparatory activity. To identify regions involved in switch-specific preparation in the mixed-dimension task block, a repeated measures ANOVA was conducted using the within-subjects factors of switch (repeat, switch within, or switch across), and MR frame (six frames: $1.5-10.5 \mathrm{sec}$ ) for each ROI separately. ROIs involved in switch-specific preparation were indicated by a significant switch $\times$ MR frame interaction (correct $p$ value: $p<.05$ divided by 39 ROIs, or $p<.00128$ ). Two planned contrasts were specified for the switch factor: (1) switch within versus repeat and (2) switch across versus switch within. These planned contrasts helped us to determine the precise nature of significant switch $\times$ MR frame interactions that were observed in several ROIs. To identify regions involved in switch-specific preparation in the single-dimension task block, the same procedure was applied, but the switch factor had just two levels: repeat and switch within. The Greenhouse-Geisser correction was applied where appropriate, and only corrected $F$ values will be reported.

Effects of global task context on switch-specific preparatory activity. To examine the effects of the overall task context in each ROI, we entered the cue-related responses for repeat trials and switch-within trials into repeated measure ANOVAs using the within-subjects factors of switch (repeat or switch within), task block (single dimension or mixed dimension), and MR frame (six frames: $1.5-10.5 \mathrm{sec}$ ). A significant interaction between switch, task block, and MR frame would indicate a difference in switch-specific preparatory activity between the single-dimension and mixed-dimension task blocks.

We also examined whether cue-only responses in repeat trials and those in switch-within trials changed (e.g., increased) across task blocks. For each ROI, we entered the cue-only responses from repeat trials and switch-within trials into separate repeated measure ANOVAs using the within-subjects factors of task block and MR frame (six frames: $1.5-10.5 \mathrm{sec}$ ). Task block had three levels (repeated, single dimension, or mixed dimension) when responses on repeat trials were compared across task blocks, but only two levels (single dimension or mixed dimension) when responses on switchwithin trials were compared across task blocks. These analyses allowed us to investigate the influence of global task context on cuerelated processes. As was described above, $p$ values were corrected for the number of ROIs in our study ( $p<.05$ divided by 39 ROIs, or $p<$ .00128 ), and the Greenhouse-Geisser correction was applied where appropriate to ensure that only corrected $F$ values would be reported. 


\section{Eye Movement Analysis}

The analysis of the EOG data during the training session was performed offline. First, the continuous data were segmented into epochs starting $100 \mathrm{msec}$ before and ending 1,450 $\mathrm{msec}$ after the cue (regardless of whether the cue was followed by a target). Next, trials during which artifacts, such as blinks, occurred were excluded from the analysis. These were identified on the vertical EOG channel, using a threshold of $\pm 40 \mu \mathrm{V}$. The remaining trials were averaged according to cue type (left, right, or color [collapsed across blue and yellow]) and task block type (repeated, single dimension, or mixed dimension), yielding a total of nine trial types. We collapsed across cues coding for blue and yellow, since both types of color cues encouraged the subjects to maintain fixation. To examine the presence of horizontal eye moments across the cue-target interval, the 1,450-msec cue-target interval was divided into 36 time bins of $40 \mathrm{msec}$ each (10 sample points) for each trial type. For each time bin, the average voltage amplitude at the horizontal EOG channel was then computed for every trial type separately. To investigate whether the subjects moved their eyes, one-sample $t$ tests were performed on these mean voltage values, separately for attend left cue trials, attend right cue trials, and attend color cue trials in the repeated, single-dimension, and mixed-dimension task blocks. The Greenhouse-Geisser correction was applied where appropriate, and only corrected $F$ values will be reported. Because of multiple interrelated comparisons and, hence, the likelihood of false-spurious significant effects, eye movement effects were considered present only if they persisted for at least 3 successive time bins [ $40 \mathrm{msec}$ each, (corrected) $p$ value $<.05$ ]

\section{RESULTS}

\section{Behavioral Data}

Behavioral performance measures are listed in Table 1. As was expected, the subjects responded $30 \mathrm{msec}$ more quickly on repeat than on switch-within trials in the singledimension task block, as reflected by a main effect of switch $[F(1,13)=12.3, p<.01]$. There was also a main effect of switch in the mixed-dimension task block $[F(2,26)=28.7, p<.001]$. Specific comparisons analyzing this effect further revealed that the subjects responded $51 \mathrm{msec}$ more quickly on repeat than on switch-within trials $(p<.05)$ and $44 \mathrm{msec}$ more quickly on repeat trials than on switch-across trials $(p<.05)$. However, response times for switch-within and switch-across trials did not significantly differ from each other. No differences in error rates were found between switch and repeat trials in the singledimension task block or in the mixed-dimension task block $(p>.05)$. Furthermore, omitted response rates did not differ between repeat and switch-within trials in the single-dimension task block. However, omitted response rates were unexpectedly somewhat lower (i.e., 1.6\%) on switch-within trials than on switch-across and repeat trials in the mixed-dimension task block $[F(2,26)=10.69$, $p<.001]$.

We also investigated whether performance in repeat and switch-within trials differed between the different task blocks. To do so, we used a repeated measures ANOVA with task block as a within-subjects factor. Neither reaction times nor error rates differed between task blocks, either for repeat or for switch-within trials $(p>.05)$. Furthermore, omitted response rates did not differ on repeat trials between the repeated, single-dimension, and mixed-dimension task blocks. However, omitted response rates were significantly lower on switch-within trials in mixed-dimension blocks than in single-dimension blocks $[F(1,13)=14.9, p<.005]$. This unexpected effect, albeit significant, was relatively small; the difference in omitted response rate between the mixed-dimension and single-dimension task blocks was, on average, $2.7 \%$ (i.e., on average, on the cue-plus-target trials, responses were recorded on $96.6 \%$ vs. $93.9 \%$ of the trials in the mixeddimension vs. the single-dimension task block). Thus, these small differences in response rate on the switchwithin cue-plus-target trials in the single-dimension versus the mixed-dimension task blocks are unlikely to explain any effects observed in the imaging data, especially for the cue-only trials.

No differences in response times or omitted response rates to catch cues were observed between the repeated (637 msec; 6.5\%), single-dimension (626 msec; 4.1\%), and mixed-dimension (639 msec; 5.8\%) task blocks. Thus, there was no evidence that cue identification processes differed across blocks.

\section{fMRI Data}

We predicted that (1) brain areas in the frontal and parietal cortex previously implicated in set switching (e.g., Wager, Jonides, \& Reading, 2004; Wager, Jonides, Smith, $\&$ Nichols, 2005) would show switch-specific preparatory activity and (2) increasing the number of possible features and dimensions within a block would reduce the magnitude of switch-specific preparatory activity in these brain areas, consistent with prior suggestions in the literature based on behavioral studies (Mayr \& Kiegl, 2003).

Effects of set switching on preparatory brain activity. In line with our first prediction, voxel-wise analyses showed that cue-related responses in a network of frontal and parietal areas previously implicated in set switching

Table 1

Performance Data: Response Times, Error Rates, and Omitted Response (OR) Rates on Repeat, Switch-Within, and/or Switch-Across Trials in the Repeated, Single-Dimension, and Mixed-Dimension Task Blocks (TBs)

\begin{tabular}{|c|c|c|c|c|c|c|}
\hline & \multirow{2}{*}{$\begin{array}{c}\text { Repeated } \\
\text { TB }\end{array}$} & \multicolumn{2}{|c|}{ Single-Dimension TB } & \multicolumn{3}{|c|}{ Mixed-Dimension TB } \\
\hline & & Repeat & Switch Within & Repeat & Switch Within & Switch Across \\
\hline Response time (msec) & 629 & 665 & 695 & 628 & 679 & 672 \\
\hline Error rate $(\%)$ & 2.7 & 3.4 & 4.2 & 4.0 & 4.5 & 3.3 \\
\hline OR rate $(\%)$ & 3.2 & 4.7 & 6.1 & 5.0 & 3.4 & 5.0 \\
\hline
\end{tabular}


changed as a function of set-switching demands (see Figure 2). Twelve ROIs showed effects of advance set switching in the single-dimension and/or mixed-dimension task blocks, as reflected by a significant interaction between the switch and the MR frame factors (see the Method section). These included the presupplementary motor area (pre-SMA; Brodmann area [BA] 6), the dorsal premotor cortex (PreMot, BA 6), the precuneus (PreCun, BA 7), the posterior intraparietal sulcus (pos IPS; BA 7), and the right fusiform gyrus (FFG; BA 19/37). See Table 2 for coordinates (column 3 ) and $F$ values (columns 4 and 5) and Figures 3 and 4 for the corresponding group BOLD signal time courses for the different cue-only trial types.

Recall that on switch-within trials, in contrast to repeat trials, both the cue identity and the cued object feature that was to be attended changed with respect to the preceding trial. Therefore, the greater cue-related activity on switchwithin trials than on repeat trials cannot unequivocally be attributed to switch-specific preparatory processes. However, in the mixed-dimension task blocks, both switchacross and switch-within trials involved (1) a cue switch and (2) a set switch. The set switch on switch-across trials, however, involved a switch of attention to one of two possible features in a different stimulus dimension, whereas the set switch on switch-within trials involved a switch of attention to the one other feature within the same stimulus dimension. Thus, greater activity in switch-across versus switch-within cue-only trials in the mixed-dimension task blocks would more convincingly indicate a role for a particular brain area in preparatory aspects of set switching. Post hoc comparisons revealed that most areas showing an advance switch effect in the mixed-dimension task block were also more strongly activated by switch-across than by switch-within cue-only trials. These areas were the left PreMot, right PreCun, and left and right pos IPS. The findings above therefore support the view that switchspecific advance preparation is possible (Meiran, Chorev, \& Sapir, 2000; Monsell, 2003; Rogers \& Monsell, 1995). Importantly, response times and error rates did not significantly differ between switch-across and switch-within trials in the mixed-dimension task block, weighing against the possibility that the difference between switch-across and switch-within cue activity was driven by a difference in the expected difficulty of the upcoming task.

Although the pre-SMA did not show a significant effect of advance set switching in the mixed-dimension task block, as reflected by an interaction between switch and MR frame, it is known to play an important role in attentional control (e.g., Weisman, Warner, \& Woldorff, 2004). Therefore, we explored the possibility that this region was more strongly activated by cues on switch-across trials than by cues on switch-within trials, using post hoc tests. As was predicted, these tests confirmed that responses in the preSMA were greater on switch-across than on switch-within cue-only trials in the mixed-dimension task block.

Effects of global task context on switch-specific preparatory brain activity. Our second prediction was that increasing the number of possible task sets (i.e., features and dimensions) that could be cued within a block would reduce the magnitude of switch-specific preparatory activity. In line with this view, the difference in activity between switch-within cue-only and repeat cue-only trials was smaller in the mixed-dimension task block than in the single-dimension task block in most brain areas showing switch-specific preparatory activity (see Figures 3 and 4, middle compared with right panel). Repeated measures ANOVAs revealed that this effect of task block on switchspecific preparatory activity was significant for the left

Table 2

Regions of Interest Showing Switch-Specific Preparatory Activity and/or Effects of Global Task Context

\begin{tabular}{|c|c|c|c|c|c|c|c|c|c|}
\hline \multirow[b]{2}{*}{ Region } & \multirow[b]{2}{*}{ Area } & $\mathrm{Coo}$ & dinates & $\mathrm{s}(\mathrm{mm})$ & \multirow{2}{*}{$\begin{array}{l}\text { Mixed-Dimension } \\
\text { Task Block } \\
\text { (Switch } \times \\
\text { MR Frame) }\end{array}$} & \multirow{2}{*}{$\begin{array}{l}\text { Single-Dimension } \\
\text { Task Block } \\
\text { (Switch } \times \\
\text { MR Frame) }\end{array}$} & \multirow{2}{*}{$\begin{array}{c}\text { Switch } \times \\
\text { Task Block } \times \\
\text { MR Frame }\end{array}$} & \multirow{2}{*}{$\begin{array}{c}\text { Repeat Trials } \\
\text { (Task Block } \times \\
\text { MR Frame) }\end{array}$} & \multirow{2}{*}{$\begin{array}{c}\text { Switch-Within } \\
\text { Trials } \\
\text { (Task Block } \times \\
\text { MR Frame) }\end{array}$} \\
\hline & & $x$ & $y$ & $z$ & & & & & \\
\hline Frontal & $\begin{array}{l}\text { Left premotor } \\
\text { Left pre-SMA } \\
\text { Right premotor }\end{array}$ & $\begin{array}{r}-30 \\
-30 \\
-4 \\
34 \\
38\end{array}$ & $\begin{array}{r}-8 \\
-4 \\
4 \\
-4 \\
-4\end{array}$ & $\begin{array}{l}49 \\
64 \\
53 \\
41 \\
41\end{array}$ & $\begin{array}{l}5.9^{* * *} \\
3.2^{* * *} \\
3.9^{* * *} \\
3.0^{* *}\end{array}$ & $\begin{array}{c}4.3^{* * *} \\
7.8^{1 * * *} \\
8.4^{* * *} \\
3.5^{* * *} \\
12.5^{1 * * *}\end{array}$ & $\begin{array}{c}3.8^{* * *} \\
7.3^{2^{*}} \\
7.2^{2^{* *}} \\
15.8^{2^{* * *}} \\
8.2^{2^{*}}\end{array}$ & $\begin{array}{l}2.6^{*} \\
2.7^{* *} \\
2.8^{* *} \\
4.1^{* * *} \\
2.9^{* *}\end{array}$ & $\begin{array}{l}4.3^{* * *} \\
3.0^{* *} \\
4.4^{* * *} \\
3.7^{* * *} \\
2.9^{* *}\end{array}$ \\
\hline Parietal & $\begin{array}{l}\text { Left precuneus } \\
\text { Left pos IPS }\end{array}$ & $\begin{array}{r}-4 \\
-26 \\
-26 \\
-26 \\
4 \\
30\end{array}$ & $\begin{array}{l}-56 \\
-60 \\
-75 \\
-53 \\
-53 \\
-60\end{array}$ & $\begin{array}{l}53 \\
49 \\
34 \\
41 \\
49 \\
49\end{array}$ & $\begin{array}{l}\quad{ }^{\dagger} \\
6.3^{* * *} \\
2.8^{* *} \\
3.5^{* * *}\end{array}$ & $\begin{array}{l}5.9^{* * * *} \\
5.5^{* * *} \\
3.9^{* * *} \\
\\
3.6^{* * *} \\
4.0^{* * *}\end{array}$ & $\begin{array}{l}4.7^{* * *} \\
22.3^{2 * * *} \\
8.4^{2 *} \\
6.0^{* * *}\end{array}$ & $\dagger$ & $\begin{array}{l}2.6^{*} \\
3.5^{* * *} \\
9.8^{* * *} \\
5.9^{* * *} \\
\\
7.9^{* * *}\end{array}$ \\
\hline Occipital & Right fusiform gyrus & 41 & -64 & -26 & & $3.5^{* * *}$ & & & \\
\hline
\end{tabular}

Note-MNI coordinates and $F$ values are listed for brain regions showing switch-specific preparatory activity in the mixed-dimension task block (column 4), switch-specific preparatory activity in the single-dimension task block (column 5), effects of task block (repeated, single dimension, mixed dimension) on cue-related responses in repeat (column 7) and switch-within (column 8) trials. Column 6 displays $F$ values for those regions of interest, in which switch-specific preparatory activity was affected by global task context. pre-SMA, presupplementary motor area; pos IPS, posterior intraparietal sulcus. ${ }^{*} p<.05 .{ }^{* *} p<.005 .{ }^{* * *} p<.05 / 39(=.0013) . \quad{ }^{\dagger} p<.1 . \quad{ }^{1}$ No interaction between switch and MR frame, but a significant main effect of switch was observed for this region. ${ }^{2}$ No interaction between switch, task block, and MR frame, but a significant interaction effect between switch and task block was observed for this region. 


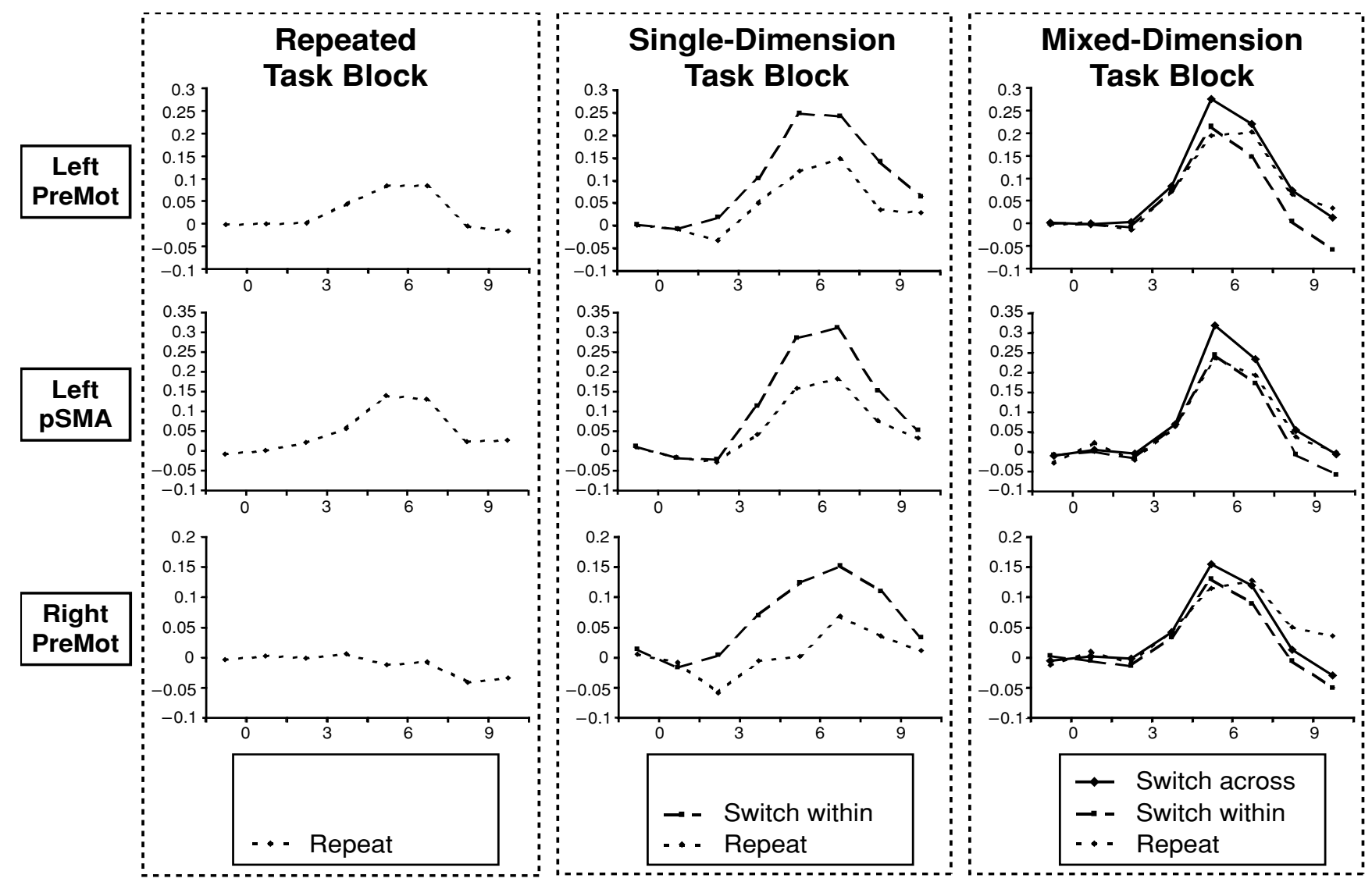

Figure 3. Group-averaged time courses in several frontal regions. Shown are group time courses for cue-related responses in repeat, switch-within, and switch-across trials in the repeated (left panel), single-dimension (middle panel), and/or mixed-dimension (right panel) task blocks. The $y$-axis is the percentage of signal change from baseline, and the $x$-axis is time in seconds. This figure clearly illustrates that cue-related responses increased not only as a function of set switching within a block of trials (middle and right panels), but also as a function of task block (i.e., the number of possibly relevant features and dimensions within a block). Note, specifically, the gradual increases in cue-related responses for repeat trials going from the repeated to the single-dimension to the mixed-dimension task blocks. PreMot, premotor cortex; pSMA, presupplementary motor area.

and right posterior IPS (i.e., $p<.0013$ ) and was nearly significant for the left and right PreMot and the left preSMA (i.e., $p<.005$; see Table 2 , column 6 , for $F$ values). These results thus demonstrate that global task context is a critical determinant of whether switch-specific preparatory activity is observed.

As can be seen in Figure 5, the effects of task block on switch-specific preparatory activity on cue-only trials were due to a selective increase in the amplitude of the responses for repeat trials in the mixed-dimension, relative to the single-dimension, task blocks. ROI analyses revealed that cue-only activity for repeat trials increased progressively from the repeated to the single-dimension to the mixed-dimension task blocks in most brain regions showing an effect of advance set switching (see Figures 3, 4, and 5A). However, repeated measure ANOVAs revealed that this increase in response amplitude across blocks achieved significance only in anterior regions (i.e., the bilateral PreMot and left pre-SMA), as indicated by significant interactions between task block and MR frame (see
Table 2 for coordinates and $F$ values, column 7). This increase in repeat trial responses is consistent with the view that increasing the number of possible task sets in a block reduces switch-specific preparatory processes (Mayr \& Kiegl, 2003). Of importance, such an increase across task blocks was not observed for switch-within trials (see Figure 5), suggesting that increasing the number of possibly relevant features and dimensions within a block may make repeat cue trials more like switch cue trials.

As we mentioned above, there were no effects of task block on the amplitude of cue-only activity for switchwithin trials. However, task block did modulate other aspects of switch-within cue-only activity in most frontal and parietal regions showing an effect of advance set switching (Table 2, column 8). Specifically, in switch-within cue-only trials, task block affected cue-related response decay, rather than response amplitude (see Figure 5). This effect was confirmed in repeated measure, random effect ANOVAs analyzing interactions between task block (single dimension or mixed dimension) and MR frame 

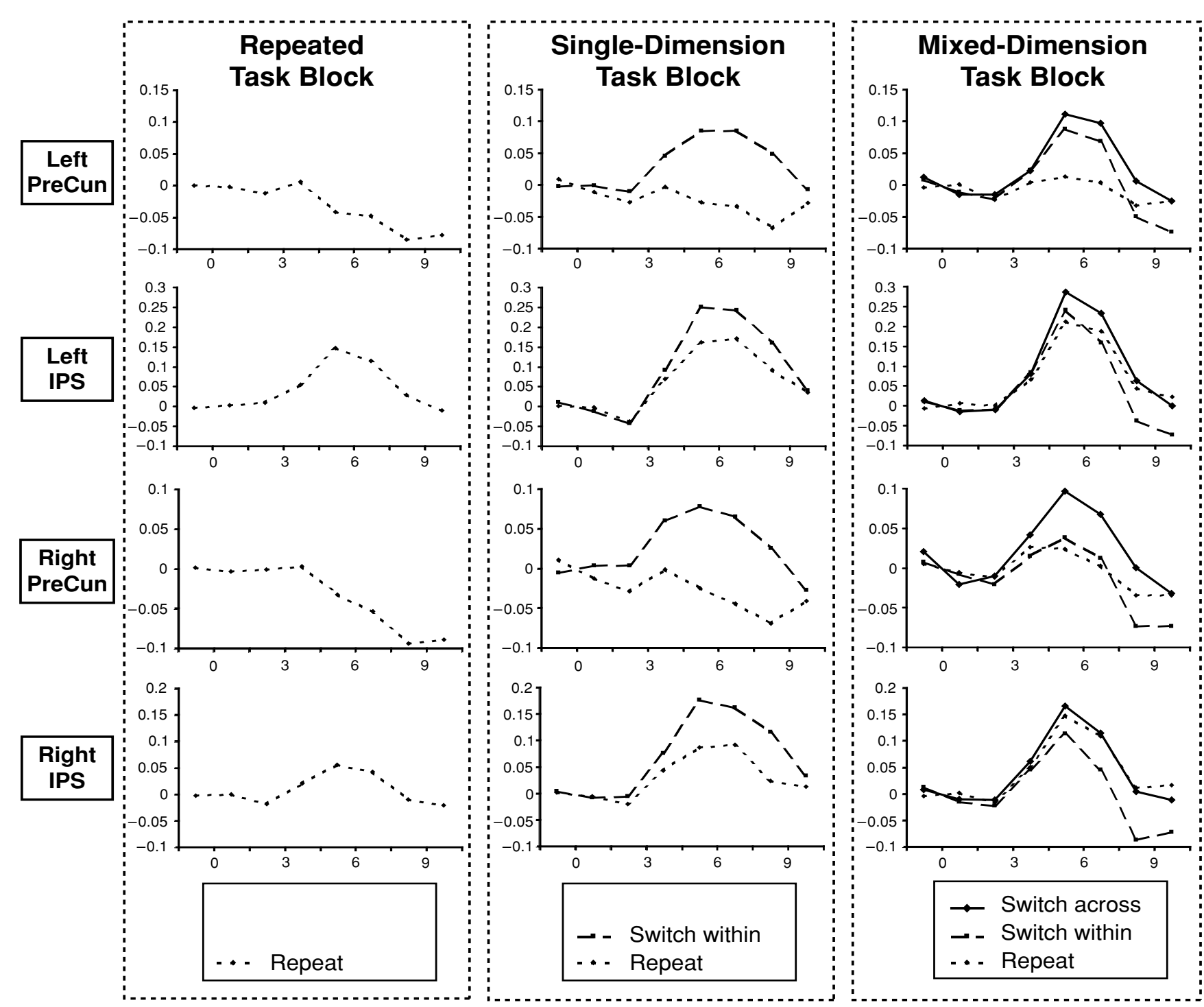

Figure 4. Group-averaged time courses in several parietal areas. Shown are time courses for cue-related responses in repeat, switchwithin, and switch-across trials in the repeated (left panel), single-dimension (middle panel), and/or mixed-dimension (right panel) task blocks in several parietal regions. The $y$-axis is the percentage of signal change from baseline, and the $x$-axis is time in seconds. This figure clearly illustrates that cue-related responses increased not only as a function of set switching within a block of trials (middle and right panels), but also as a function of task block (i.e., the number of possibly relevant features and dimensions within a block). Note, specifically, the gradual increases in cue-related response for repeat trials going from the repeated to the single-dimension to the mixed-dimension task blocks. PreCun, precuneus; IPS, posterior intraparietal sulcus.

(three frames: peak frame plus two subsequent frames). Significant interactions in these ANOVAs indicated faster hemodynamic response decay on switch-within trials in the mixed-dimension versus the single-dimension task blocks in the bilateral PreMot and left pos IPS $(p<.05$; see Figure 5B). Similarly, in the mixed-dimension task block cue-only responses on switch-across trials decayed significantly more quickly than responses on repeat trials $(p<.05)$ in these same brain areas (i.e., the bilateral PreMot and left pos IPS; see Figures 3 and 4, right panels). In these areas, hemodynamic response decay in the mixed- dimension task block was, thus, generally faster for switch cue-only than for repeat cue-only trials.

\section{Eye Movements}

Analyses of the cue-related EOG signal revealed that the subjects did not move their eyes appreciably in any of the task blocks during the training session, since the cue-related EOG signal did not significantly deviate from zero in any of the tested conditions. In a previous study from our group (Giesbrecht, Woldorff, Song, \& Mangun, 2003), the same peripheral stimuli were used as in 


\section{A. Effects of Task Block on Response Amplitude}
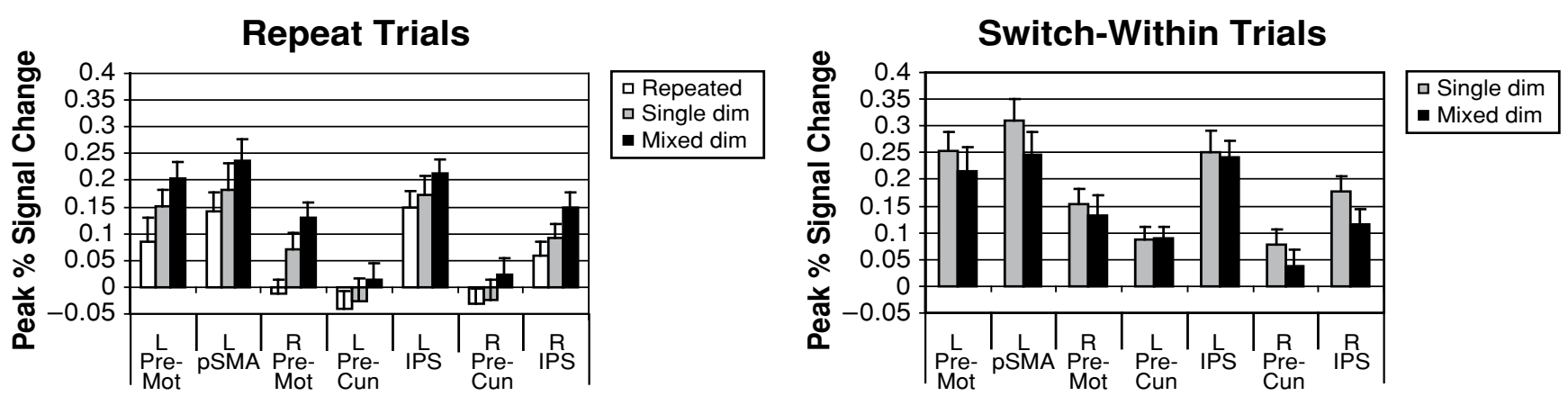

\section{B. Effects of Task Block on Response Decay on Switch-Within Trials}



Figure 5. (A) Cue-related peak percentage of signal change values (plus standard error) for frontal and parietal areas showing switch-specific preparatory activity. Shown are time courses for repeat (left column figure) and switch-within (right column figure) trials in the repeated, single-dimension, and/or mixed-dimension task blocks. Note how activation amplitudes on repeat trials generally increased as a function of task block, whereas activation amplitudes on switch-within trials were relatively unaffected by the type of task block in which they were presented. (B) Group time courses for switch-within trials presented in the single-dimension and mixeddimension task blocks for the same areas as those shown in panel $A$. The $y$-axis is the percentage of signal change from baseline, and the $\boldsymbol{x}$-axis is time in seconds. Note how cue-related responses decayed more quickly on switch-within trials in the single-dimension task block than in the mixed-dimension task block. Single-Dim, single dimension; Mixed-Dim, mixed dimension; L, left; R, right; Front, frontal; Par, parietal; PreMot, premotor cortex; pSMA, presupplementary motor area; PreCun, precuneus; IPS, intraparietal sulcus.

the present study. Using a calibration task, they estimated voltage fluctuations produced by voluntary eye movements to these peripheral stimuli and found that instructed horizontal eye movements of $1^{\circ}$ of visual angle elicited a response of about $10 \mu \mathrm{V}$. In the present study, the deviation in horizontal EOG amplitude in the training session on left cue trials or on right cue trials was never more than $\pm 0.6 \mu \mathrm{V}$. This result indicates that the subjects did not move their eyes during the cue-target interval in the training session and, therefore, that eye movements are unlikely to account for the differential cue-related activity we observed.

\section{DISCUSSION}

The present study had two main goals. The first was to resolve a long-standing debate concerning whether it is possible to prepare in advance to switch to perform- 
ing a new task. On the basis of behavioral data, in which cue-related preparatory processes are expressed only indirectly, some investigators have argued that the decrease in response time on set switch trials with longer preparation intervals reflects preparatory processes that enable set switching (Meiran, 1996; Rogers \& Monsell, 1995). Other behavioral studies, however, have suggested that this preparation effect reflects the additional time given to encode a new instructional cue, regardless of whether a set switch occurs (Altmann, 2004; Logan \& Bundesen, 2003). The present study aimed at resolving this issue by using event-related fMRI as a more direct measure of cuerelated, switch-specific preparatory processes. The second goal of this study was to determine the effects of global task context on brain activity associated with switchspecific task preparation.

There were two central findings. First, several frontal and parietal regions typically associated with cognitive control exhibited switch-specific preparatory activity. These effects provide direct support for the view that it is possible to prepare in advance for an upcoming task switch (Meiran et al., 2000; Rogers \& Monsell, 1995). In addition, they indicate which brain areas are involved in preparatory aspects of switching between different attentional sets and, moreover, that these areas involve parts of the frontoparietal control network. Second, as was predicted, increasing the number of possibly relevant features and dimensions within a block reduced the magnitude of switch-specific preparatory activity. This effect was due to a selective increase in cue-related activity on repeat trials, since the number of possible task sets that could be cued within a block increased across the repeated, single-dimension, and mixed-dimension task blocks. This novel finding is in line with behavioral data indicating that global task context affects the ability to observe switchspecific task preparation effects in response time (Mayr \& Kiegl, 2003). Moreover, it may explain why previous fMRI studies generally did not show any differences in cue-related brain activity between switch and repeat trials (Brass \& von Cramon, 2002; Luks et al., 2002; Ruge et al., 2005; Shulman et al., 2002). Indeed, our findings suggest that having to switch between multiple task sets in those studies might have reduced the probability of observing preparatory switch-related activity.

\section{Switch-Specific Preparatory Activity in the Frontoparietal Network}

Switch-specific anticipatory activity was observed in the present study in several frontal and parietal brain areas previously implicated in set switching (Wager et al., 2004) and is consistent with behavioral findings suggesting that it is possible to prepare for an upcoming set switch (Meiran, 1996; Rogers \& Monsell, 1995). Of importance, the present findings also delineate more precisely the nature of switch-specific activity that has been observed in previous fMRI studies. For example, several studies have isolated switch-specific activity averaged across cue and target periods (Kimberg et al., 2000; Smith et al., 2004) or by comparing separate blocks of set switch and set repeat trials (Shulman et al., 2002; Sohn et al., 2000). Together with the results from a recent event-related fMRI study (Barber \& Carter, 2005), our findings, which are based on cue-specific activity, provide direct evidence implicating specific brain areas in switch-specific task preparation.

Brain areas exhibiting switch-specific preparatory activity included the pre-SMA, the dorsal PreMot, the posterior part of the IPS, the PreCun, and the right FFG. All of these areas displayed significantly greater cue-related activity on switch-within trials than on repeat trials. In addition, most of these areas also exhibited greater cuerelated activity on switch-across than on switch-within trials in the mixed-dimension task block. In switch-across and switch-within trials, both the cue and the cued object changed with respect to the preceding trial. Therefore, the greater cue-related activity on switch-across than on switch-within trials could not have been due to the need to encode a new instructional cue (Altmann, 2004; Logan \& Bundesen, 2003; Mayr \& Kliegl, 2003). Rather, it was more likely specific to preparatory processes related to set switching. That is, on switch-across trials, the subjects had to switch their attention to one of two possible features in a different stimulus dimension, whereas on switch-within trials they needed only to switch their attention to the one other feature within the same stimulus dimension. Brain regions showing greater preparatory activity on switchacross than on switch-within trials included the left and right pos IPS, the right PreCun, the pre-SMA, and the left PreMot. As will be discussed next, these regions may act as a network of communicating areas, each mediating a different subprocess related to preparing for a set switch.

In the parietal cortex, switch-specific preparatory activity was localized to the IPS and PreCun. The posterior part of the IPS has been associated with attention-in particular, attention shifting (Le, Pardo, \& Hu, 1998; Liu, Slotnick, Serences, \& Yantis, 2003; Shulman et al., 2002; Vanderberghe, Gitelman, Parrish, \& Mesulam, 2001; Yantis et al., 2002). The functional role of the PreCun in set switching, however, is less well understood. In the recent study by Barber and Carter (2005), PreCun activity was associated with both overcoming prepotent response tendencies and switching between task sets. Indeed, many tasks requiring response selection activate this area (Banich et al., 2001; Dove, Pollmann, Schubert, Wiggins, \& von Cramon, 2000; Schumacher, Puni, and D'Esposito, 2003), as do tasks requiring shifts of attention in space (Corbetta, Miezin, Shulman, \& Petersen, 1993; Posner, Walker, Friedrich, \& Rafal, 1984) and tasks of working memory (LaBar, Gitelman, Parrish, \& Mesulam, 1999). Set switching involves processes related to attention, working memory, and response selection. Thus, further studies will be needed to determine the specific contribution of the PreCun to set switching.

In the frontal cortex, the effects of advance set switching were confined to dorsal PreMot regions and to the pre-SMA. More lateral dorsal PreMot regions have recently been implicated in the analysis and interpretation 
of the functional significance of the cue symbol, whereas more medial dorsal PreMot areas have been more specifically associated with the orienting of attention (Woldorff et al., 2004). In the present study, advance set switching affected dorsal PreMot areas close to the medial PreMot areas identified in Woldorff et al. (2004). Set switching may thus increase demands on processes that focus attention on the relevant stimulus attribute. As for the preSMA, this region has previously been implicated both in set switching (Dove et al., 2000; Rushworth et al., 2001) and in preparatory processing (Brass \& von Cramon, 2002; Luks et al., 2002; Weissman et al., 2004). Studies in monkeys (Hoshi \& Tanji, 2004; Sakai et al., 1999) and humans (Picard \& Strick, 2001) have indicated that the pre-SMA is involved in transforming sensory information into information required for motor planning. Within the present context, this transformation process may have been more demanding on switch trials than on repeat trials, since the stimulus-response mappings changed only on switch trials.

An interesting aspect of our study is the lack of preparatory activation in frontal areas anterior to the PreMot cortex. Lateral prefrontal areas have been implicated in advance preparation in some (Brass \& von Cramon, 2002; Luks et al., 2002; Shulman et al., 2002), albeit not all (Barber \& Carter, 2005), previous fMRI studies. The lack of cue-related prefrontal activation may indicate that recruitment of prefrontal areas is dependent on the type of task used to study advance set switching. For instance, the prefrontal cortex may be involved only when more complex tasks are used that require active maintenance and/or manipulation of information in working memory (Corbetta \& Shulman, 2002). In line with this suggestion, a recent meta-analysis demonstrated that executive working memory processes, but not set switching, recruited anterior frontal and dorsolateral prefrontal areas (Wager et al., 2004). Of importance, the task used in the present study was relatively simple, in comparison with previous fMRI studies of advance preparation reporting prefrontal activation. For example, some investigators used relatively more complex tasks in which subjects had to indicate whether a target number was greater or smaller than a specific number or was odd or even (Brass \& von Cramon, 2002, 2004; Luks et al., 2002). These computationally more demanding tasks likely increased demands on prefrontal areas involved in executive processing. It is noteworthy in this respect that, as in the present study, frontal activations reported in attentional-cuing studies are usually confined to more posterior frontal regions (Corbetta \& Shulman, 2002; Giesbrecht \& Mangun, 2005; Woldorff et al., 2004).

A second reason why we may not have observed prefrontal activation is that we used relatively short intervals between cues and target stimuli $(1.5 \mathrm{sec})$, in comparison with prior fMRI studies (e.g., $8 \mathrm{sec}$ in Hopfinger, Buonocore, \& Mangun, 2000, 2.5-5 sec in Luks et al., 2002, and $3.8 \mathrm{sec}$ in Shulman et al., 2002). As was noted by Woldorff et al. (2004), longer delays between cues and target stimuli may increase demands on working memory maintenance processes and, hence, lead to greater prefrontal activity. Thus, the present findings support the view that prefrontal activity is reduced when (1) the task is relatively simple and/or (2) the period between the instructional cue and the target is not so extended in time.

Switch-specific activity was also observed in the right FFG. Here, there was greater cue-related activity on switch-within than on repeat trials, but not on switchacross trials in comparison with switch-within trials. As was mentioned earlier, in both switch-within and switchacross trials, the cue stimulus changed with respect to the preceding trial, whereas in repeat trials, the cue stimulus remained the same. Thus, the pattern of activity in the right FFG is consistent with a role in this region in encoding a new (i.e., different from the preceding trial) instructional cue. In line with this possibility, the right FFG is known to be involved in the encoding of visual objects, such as faces (e.g., Kanwisher, McDermott, \& Chun, 1997). Moreover, Brass and von Cramon (2004) have also recently suggested that the FFG plays a role in cue encoding.

\section{Modulatory Effects of the Global Task Context on Advance Set Switching}

Several previous investigators have suggested that it may become more difficult to recruit switch-specific preparatory processes as the number of possible task sets in a block increases (Mayr \& Kiegl, 2003). In line with this hypothesis, the difference in peak activity between switch-within and repeat cue-only trials was smaller in the mixed-dimension than in the single-dimension task block in all frontal and parietal regions involved in advance set switching. However, this effect was due mainly to a selective increase in cue-related activity on repeat trials, since the number of possibly relevant features and/or dimensions within a block increased across the repeated, single-dimension, and mixed-dimension task blocks. Interestingly, for switch-within cue-only trials, increasing the number of possibly relevant features and dimensions affected cue-related response decay, but not response amplitude, in several frontal and parietal brain regions. While the precise functional significance of this time course effect is not known, a similar observation of differential response decay has been made by Braver et al. (2003), although these authors did not separate cue- from targetrelated activity.

Although the effects of global task context on switchspecific preparatory activity were highly robust in our study, there are several hypotheses to consider regarding the precise cause of this effect. The repeated, singledimension, and mixed-dimension task blocks differed in both the number of task sets and the probability of having to switch between sets. Both of these contextual factors may have modulated the recruitment of cue-triggered executive processes underlying set switching. For example, it is possible that the cue interpretation and cue-to-set mappings become too difficult to maintain in working memory as the 
number of possible features and/or perceptual dimensions in a trial block increases, so that subjects need to drop the previous task set in order to successfully perform this mapping selection. If this were true, however, it would seem that greater demands on processes that link a task cue to a particular task set should increase activity for all types of cues, rather than only repeat cues, as we observed.

A second possibility is that subjects strategically disengage from the task set used in the previous trial when there are more possible sets and the probability of a set switch in the next trial is high. Such a strategy would lead to selective increases in cue-related activity for repeat cues, as we observed, since a repeat cue may tend to be processed more like a switch cue if subjects disengage from the task set used in the previous trial. In line with this possibility, when subjects experience greater uncertainty about the category of a forthcoming event, they may abandon category-specific preparation (Los, 1996). However, it has also been shown that disengagement from the previous task set is more likely when subjects are given additional information about the identity of the actual upcoming task to which they need to switch (Dreisbach, Haider, \& Kluwe, 2002). In the present study, the subjects may have expected a switch more often in the mixed-dimension task block, but they did not know the rectangle to which they would need to switch their attention. Thus, it is not clear that a strategic disengagement of the task set used in each trial can explain the selective increases of activity for repeat cues observed in the present study. Future studies are needed to fully evaluate this possibility.

A third possible explanation for the effects of global task structure on switch-specific preparatory activity is that increasing the number of possible features and dimensions in a trial block leads subjects to adopt different global control strategies. It has been argued that global control strategies affect the way in which local control requirements (e.g., a task transition) are handled (Gopher, Armony, \& Greenspan, 2000; Strayer \& Kramer, 1994). Indeed, at the behavioral level, there is growing evidence that switch costs depend not only on the set switch actually performed, but also on the representational structures in which the individual tasks are embedded (Kleinsorge \& Heuer, 1999; Kleinsorge, Heuer, \& Schmidtke, 2004; Lien \& Ruthruff, 2004). For example, in the present study, the subjects may have integrated the individual features (i.e., blue, yellow, left, and right) at the dimension level in the mixeddimension task block (e.g., blue and yellow were considered the same task), while representing the individual features (e.g., blue and yellow) separately in the single-dimension task block. Future studies are necessary to more precisely determine how the number of possible task sets in a block and/or the probability of switching between sets may affect switch-specific preparatory processes.

\section{Interpreting Switch-Specific Preparatory Activity}

One may wonder whether the switch-specific preparatory activity that we observed reflects processes specific to set switching (Monsell, 2003; Rogers \& Monsell, 1995) or, alternatively, reflects additional recruitment of generalized task preparation processes that are triggered for all trials (Altmann, 2004). In the present study, many of the brain areas showing switch-specific preparatory activity were also activated by repeat cues. This suggests that preparation for a set switch involves recruiting many of the same basic preparatory processes as those involved in preparation for a set repetition (albeit more strongly) and does not exclusively involve a set of control processes that are recruited only during endogenous task reconfiguration (Altmann, 2004). The fact that cue-related activity in repeat trials increased across task blocks in the same brain areas showing switch-specific preparatory activity provides further support for this view. Thus, the present findings suggest that at least some of the cue-related switchspecific activity we observed reflects greater recruitment of generalized task preparation processes.

\section{Conclusion}

In conclusion, we have isolated switch-specific preparatory activity in a network of dorsal frontal and parietal brain areas that are typically associated with cognitive control processes. Our data, therefore, provide direct support for the view that advance preparation for an upcoming set switch is possible. Moreover, they indicate that increasing the number of possible task sets in a trial block reduces switch-specific preparatory activity. Thus, global task structure appears to be a critical determinant of whether switch-specific preparatory activity is observed.

\section{REFERENCES}

Allport, D. A., Styles, E. A., \& Hsieh, S. (1994). Shifting intentional set: Exploring the dynamic control of tasks. In C. Ultimà \& M. Moscovitch (Eds.), Attention and Performance XV (pp. 421-452). Hillsdale, NJ: Erlbaum.

Altmann, E. M. (2003). Task switching and the pied homunculus: Where are we being led? Trends in Cognitive Sciences, 7, 340-341.

Altmann, E. M. (2004). Advance preparation in task switching. Psychological Science, 15, 616-622.

Baddeley, A. (1986). Working memory. New York: Oxford University Press.

Banich, M. T., Milham, M. P., Jacobson, B. L., WebB, A., Wszalek, T., Cohen, N. J., \& Kramer, A. F. (2001). Attentional selection and the processing of task-irrelevant information: Insights from fMRI examinations of the Stroop task. Progress in Brain Research, 134, 459-470.

Barber, A. D., \& Carter, C. S. (2005). Cognitive control involved in overcoming prepotent response tendencies and switching between tasks. Cerebral Cortex, 15, 899-912.

Brass, M., \& VON CRAMON, D. Y. (2002). The role of the frontal cortex in task preparation. Cerebral Cortex, 12, 908-914.

Brass, M., \& vON CRAMON, D. Y. (2004). Decomposing components of task preparation with functional magnetic resonance imaging. Journal of Cognitive Neuroscience, 16, 609-620.

Braver, T. S., Reynolds, J. R., \& Donaldson, D. I. (2003). Neural mechanisms of transient and sustained cognitive control during task switching. Neuron, 39, 713-726.

Buckner, R. L., Goodman, J., Burock, M., Rotte, M., Koutstaal, W., SCHACTER, D., ET AL. (1998). Functional-anatomic correlates of object priming in humans revealed by rapid presentation event-related fMRI. Neuron, 20, 285-296.

Burock, M. A., Buckner, R. L., Woldorff, M. G., Rosen, B. R., \& 
Dale, A. M. (1998). Randomized event-related experimental designs allow for extremely rapid presentation rates using functional MRI. NeuroReport, 9, 3735-3739.

Corbetta, M., Kincade, J. M., Ollinger, J. M., McAvoy, M. P., \& SHULMAN, G. L. (2000). Voluntary orienting is dissociated from target detection in human posterior parietal cortex. Nature Neuroscience, 3, 292-297.

Corbetta, M., Miezin, F. M., Shulman, G. L., \& Petersen, S. E. (1993). A PET study of visuospatial attention. Journal of Neuroscience, 13, 1202-1226.

Corbetta, M., \& Shulman, G. L. (2002). Control of goal-directed and stimulus-driven attention in the brain. Nature Reviews Neuroscience, 3, 201-215.

Dove, A., Pollmann, S., Schubert, T., Wiggins, C. J., \& von CraMON, D. Y. (2000). Prefrontal cortex activation in task switching: An event-related fMRI study. Brain Research: Cognitive Brain Research, 9, 103-109.

Dreher, J. C., Koechlin, E., Ali, S. O., \& Grafman, J. (2002). The roles of timing and task order during task switching. NeuroImage, 17, 95-109.

Dreisbach, G., Haider, H., \& Kluwe, R. H. (2002). Preparatory processes in the task-switching paradigm: Evidence from the use of probability cues. Journal of Experimental Psychology: Learning, Memory, \& Cognition, 28, 468-483.

Forstmann, B. U., Brass, M., Koch, I., \& von Cramon, D. Y. (2005). Internally generated and directly cued task sets: An investigation with fMRI. Neuropsychologia, 43, 943-952.

Friston, K. J., Holmes, A. P., Worsley, K. J., Poline, J. P., Frith, C. D., \& Frackowiak, R. S. J. (1995). Statistical parametric maps in functional imaging: A general linear approach. Human Brain Mapping, 2, 189-210.

Giesbrecht, B., \& MANGUN, G. R. (2005). Identifying the neural systems of top-down attentional control: A meta-analytic approach. In L. Itti, G. Rees, \& J. Tsotsos (Eds.), Neurobiology of attention (pp. 6368). New York: Academic Press/Elsevier.

Giesbrecht, B., Woldorff, M. G., Song, A. W., \& Mangun, G. R. (2003). Neural mechanisms of top-down control during spatial and feature attention. NeuroImage, 19, 496-512.

Gopher, D., Armony, L., \& Greenspan, Y. (2000). Switching tasks and attention policies. Journal of Experimental Psychology: General, 129, 308-339.

Hopfinger, J. B., Buonocore, M. H., \& Mangun, G. R. (2000). The neural mechanisms of top-down attentional control. Nature Neuroscience, 3, 284-291.

Hoshi, E., \& TANJI, J. (2004). Differential roles of neuronal activity in the supplementary and presupplementary motor areas: From information retrieval to motor planning and execution. Journal of Neurophysiology, 92, 3482-3499.

Kanwisher, N., McDermott, J., \& Chun, M. M. (1997). The fusiform face area: A module in human extrastriate cortex specialized for face processing. Journal of Neuroscience, 17, 4302-4311.

Kimberg, D. Y., Aguirre, G. K., \& D'Esposito, M. (2000). Modulation of task-related neural activity in task-switching: An fMRI study. Brain Research: Cognitive Brain Research, 10, 189-196.

KLEINSORge, T., \& Heuer, H. (1999). Hierarchical switching in a multidimensional task space. Psychological Research, 62, 300-312.

Kleinsorge, T., Heuer, H., \& SchmidtKe, V. (2004). Assembling a task space: Global determination of local shift costs. Psychological Research, 68, 31-40.

LaBar, K. S., Gitelman, D. R., Parrish, T. B., \& Mesulam, M. (1999). Neuroanatomic overlap of working memory and spatial attentional networks: A functional MRI comparison within subjects. NeuroImage, 10, 695-704.

LE, T. H., PARdo, J. V., \& Hu, X. (1998). 4 T-fMRI study of nonspatial shifting of selective attention: Cerebellar and parietal contributions. Journal of Neurophysiology, 79, 1535-1548.

Lien, M. C., \& Ruthruff, E. (2004). Task switching in a hierarchical task structure: Evidence for the fragility of the task repetition benefit. Journal of Experimental Psychology: Learning, Memory, \& Cognition, 30, 697-713.

Liu, T., Slotnick, S. D., Serences, J. T., \& Yantis, S. (2003). Cortical mechanisms of feature-based attentional control. Cerebral Cortex, 13, 1334-1343.

Logan, G. D., \& Bundesen, C. (2003). Clever homunculus: Is there an endogenous act of control in the explicit task-cuing procedure? Journal of Experimental Psychology: Human Perception \& Performance, 29, 575-599.

Los, S. A. (1996). On the origin of mixing costs: Exploring information processing in pure and mixed blocks of trials. Acta Psychologia, 94, $145-188$

Luks, T. L., Simpson, G. V., Feiwell, R. J., \& Miller, W. L. (2002). Evidence for anterior cingulate cortex involvement in monitoring preparatory attentional set. NeuroImage, 17, 792-802.

MaYr, U., \& KeEle, S. W. (2000). Changing internal contraints of action: The role of backward inhibition. Journal of Experimental Psychology: General, 129, 4-26.

Mayr, U., \& Kliegl, R. (2003). Differential effects of cue changes and task changes on task-set selection costs. Journal of Experimental Psychology: Learning, Memory, \& Cognition, 29, 362-372.

MeIRan, N. (1996). Reconfiguration of processing mode prior to task performance. Journal of Experimental Psychology: Learning, Memory, \& Cognition, 22, 1423-1442.

Meiran, N., Chorev, Z., \& SAPIR, A. (2000). Component processes in task switching. Cognitive Psychology, 41, 211-253.

Monsell, S. (2003). Task switching. Trends in Cognitive Sciences, 7 , 134-140.

Norman, D., \& Shallice, T. (1986). Attention to action: Willed and automatic control of behavior. In R. Davidson, G. Schwartz, \& D. Shapiro (Eds.), Consciousness and self-regulation: Advances in research and theory (Vol. 4, pp. 1-18). New York: Plenum.

PiCARD, N., \& STRICK, P. L. (2001). Imaging the premotor areas. Current Opinion in Neurobiology, 11, 663-672.

Posner, M. I., Walker, J. A., Friedrich, F. J., \& Rafal, R. D. (1984). Effects of parietal injury on covert orienting of attention. Journal of Neuroscience, 4, 1863-1874.

Rogers, R. D., \& Monsell, S. (1995). The costs of a predictable switch between simple cognitive tasks. Journal of Experimental Psychology: General, 124, 207-231.

Ruge, H., Brass, M., Koch, I., Rubin, O., Meiran, N., \& von CraMON, D. Y. (2005). Advance preparation and stimulus-induced interference in cued task switching: Further insights from BOLD fMRI. Neuropsychologia, 43, 340-355.

Rushworth, M. F., Paus, T., \& Sipila, P. K. (2001). Attention systems and the organization of the human parietal cortex. Journal of Neuroscience, 21, 5262-5271.

Sakai, K., Hikosaka, O., Miyauchi, S., Sasaki, Y., Fujimaki, N., \& Putz, B. (1999). Presupplementary motor area activation during sequence learning reflects visuo-motor association. Journal of Neuroscience, 19, RC1.

Schumacher, E. H., Puni, A., \& D'Esposito, M. (2003). Neural evidence for representation-specific response selection. Journal of Cognitive Neuroscience, 15, 1111-1121.

Shulman, G. L., D’Avossa, G., Tansy, A. P., \& Corbetta, M. (2002). Two attentional processes in the parietal lobe. Cerebral Cortex, 12, 1124-1131.

Shulman, G. L., Ollinger, J. M., Akbudak, E., Conturo, T. E., SnyDer, A. Z., Petersen, S. E., \& Corbetta, M. (1999). Areas involved in encoding and applying directional expectations to moving objects. Journal of Neuroscience, 19, 9480-9496.

Smith, A. B., TaYlor, E., Brammer, M., \& Rubia, K. (2004). Neural correlates of switching set as measured in fast, event-related functional magnetic resonance imaging. Human Brain Mapping, 21, 247-256.

Snedecor, G. W., \& Cochran, W. G. (1989). Statistical methods (8th ed.). Ames: Iowa State University Press.

Sohn, M. H., Ursu, S., Anderson, J. R., Stenger, V. A., \& Carter, C. S. (2000). Inaugural article: The role of prefrontal cortex and posterior parietal cortex in task switching. Proceedings of the National Academy of Sciences, 97, 13448-13453.

Strayer, D. L., Kramer, A. F. (1994). Strategies and automaticity: 1. Basic findings and conceptual-framework. Journal of Experimental Psychology: Learning, Memory, \& Cognition, 20, 318-341.

Vandenberghe, R., Gitelman, D. R., Parrish, T. B., \& Mesulam, 
M. M. (2001). Functional specificity of superior parietal mediation of spatial shifting. NeuroImage, 14, 661-673.

WAGER, T. D., Jonides, J., \& READING, S. (2004). Neuroimaging studies of shifting attention: A meta-analysis. NeuroImage, 22, 1679-1693.

Wager, T. D., Jonides, J., Smith, E. E., \& Nichols, T. E. (2005). Toward a taxonomy of attention shifting: Individual differences in fMRI during multiple shift types. Cognitive, Affective, \& Behavioral Neuroscience, $\mathbf{5}, 127-143$.

Weissman, D. H., Mangun, G. R., \& Woldorff, M. G. (2002). A role for top-down attentional orienting during interference between global and local aspects of hierarchical stimuli. NeuroImage, 17, 1266-1276.

Weissman, D. H., Warner, L., \& WoldorfF, M. G. (2004). The neural mechanisms for minimizing cross-modal distraction. Journal of Neuroscience, 24, 10941-10949.
WoldorfF, M. G. (1993). Distortion of ERP averages due to overlap from temporally adjacent ERPs: Analysis and correction. Psychophysiology, 30, 98-119.

Woldorff, M. G., Hazlett, C. J., Fichtenholtz, H. M., WeissMAN, D. H., Dale, A. M., \& SonG, A. W. (2004). Functional parcellation of attentional control regions of the brain. Journal of Cognitive Neuroscience, 16, 149-165.

Yantis, S., Schwarzbach, J., Serences, J. T., Carlson, R. L., Steinmetz, M. A., Pekar, J. J., \& Courtney, S. M. (2002). Transient neural activity in human parietal cortex during spatial attention shifts. Nature Neuroscience, 5, 995-1002.

(Manuscript received September 13, 2005; revision accepted for publication March 17, 2006.) 